\title{
Los límites entre actancia y la circunstancia en la Nueva gramática de la lengua española*
}

\author{
Celia Berná Sicilia ${ }^{* *}$ \\ Universidad Católica San Antonio, España
}

\section{INTRODUCCIÓN}

La distinción binaria entre los distintos elementos que componen el predicado verbal ha sido asumida de manera general en las diferentes corrientes lingüísticas, pero surge fundamentalmente a partir de las aportaciones realizadas por Tesnière ([1959] 1994) donde se inaugura un nuevo modelo teórico: la Teoría de la Valencia (en adelante, TV). Desde que Tesnière (1994) estableciera que los verbos poseían una determinada valencia o un potencial combinatorio específico configurado por diferentes actantes, el interés en buena parte de la investigación lingüística actual se ha centrado en tratar de delimitar adecuadamente qué elementos integrarían el conjunto de componentes valenciales o argumentales exigidos por el núcleo verbal que conforma un predicado.

* Trabajo integrado en el proyecto del Ministerio de Ciencia e Innovación del Gobierno de España FFI2010-19946, que lleva por título "Clases verbales y alternancias en la estructura argumental: aplicaciones a español como lengua extranjera/español como lengua segunda", desarrollado en la Universidad de Alicante, a cargo de José Luis Cifuentes Honrubia.

** Para correspondencia dirigirse a: Celia Berná Sicilia (cberna@ucam.edu), Universidad Católica San Antonio (UCAM), Facultad de Ciencias Sociales y de la Comunicación, Departamento de Periodismo, Campus de los Jerónimos, s/n Guadalupe 30107 (Murcia) España. 
La discusión en torno a la distinción entre actantes (argumentos) y circunstantes (adjuntos) viene ocupando un lugar central dentro de la reflexión lingüística, debido fundamentalmente a las insuficiencias que muestra la dicotomía transitivo-intransitivo para dar cuenta de la enorme complejidad del fenómeno lingüístico de la combinatoria verbal ${ }^{1}$. Una adecuada identificación de los componentes valenciales nos permitirá profundizar en los mecanismos esenciales que intervienen en la operación verbal y nos ayudará, a su vez, a predecir, en cierta medida, el comportamiento verbal, lo que redundará en que podamos describir, con más precisión, el lenguaje y articular mejor su enseñanza.

A la hora de abordar la diferenciación que existe entre los componentes actanciales de la cláusula y los circunstanciales, distintas son las perspectivas que se han adoptado sin que haya sido posible establecer criterios suficientemente sólidos e inequívocos como para lograr fijar con claridad y contundencia una frontera nítida entre actancia y circunstancia. Un buen reflejo de la indefinición que ha caracterizado la delimitación de los actantes en la literatura especializada es la Nueva gramática de la lengua española (en adelante, NGLE). La nueva preceptiva gramatical de la Academia (RAE 2009) incorpora, por primera vez, los planteamientos tesnerianos, pero se muestra incapaz de establecer, en consonancia con la falta de acuerdo existente en el ámbito investigador, una definición cabal de los componentes valenciales que permita identificarlos sin ningún género de dudas en el marco predicacional ${ }^{2}$.

Este trabajo tratará de ofrecer una revisión general de la diferenciación actante-circunstante que emana de la NGLE (RAE: 2009), vinculando los planteamientos del tratado académico con los que se han derivado de la reflexión en la Teoría de la Valencia desde que Tesnière publicara su obra Elementos de sintaxis estructural en 1959. El objetivo no es otro que el de proporcionar una fijación más ajustada de los límites valenciales y facilitar una definición más precisa de los componentes actanciales.

1 Las limitaciones de la dicotomía transitivo-intransitivo han sido puestas de manifiesto de manera recurrente en la literatura especializada a través de diversos trabajos, entre los que destacamos los de Perlmutter (1978), Hopper y Thompson (1980), Cano (1981), Bossong (1998) y Mendikoetxea (1999).

2 La problemática en torno a los límites de actancia y circunstancia constituye, desde que Tesniére deslindara actantes y circunstantes, uno de los puntos de mayor discusión entre los investigadores del ámbito de la gramática de valencias o de dependencias, sin que haya sido posible llegar a una solución definitiva capaz de establecer un consenso unánime en el marco de la investigación de la TV. 


\section{MARCO TEÓRICO: LA TV}

Antes de emprender el análisis de las consideraciones que la NGLE (RAE: 2009) vierte en torno a la distinción entre argumentos y adjuntos, vamos a efectuar un breve repaso por las reflexiones que, en el marco de la TV, se han realizado a propósito de cuáles son los componentes que deben integrar la órbita valencial, con objeto de que podamos ver la evolución que ha seguido esta idea en el pensamiento gramatical a lo largo del tiempo y así enjuiciar y valorar con mayor rigor cuál es la aportación que realiza la Academia en relación con esta materia en la nueva preceptiva gramatical.

Tal como hemos apuntado anteriormente, la delimitación de las fronteras entre actancia y circunstancia constituye uno de los aspectos más controvertidos en la investigación lingüística actual, hasta el punto de que puede ser considerado como un verdadero agujero negro dentro la reflexión lingüística, en general, y en el marco de la TV, en particular.

Las diversas corrientes teóricas que han abordado la cuestión valencial han adoptado posturas que fluctúan desde la estricta separación entre actantes y circunstantes, pasando por la diferenciación gradual, hasta llegar al extremo de considerar la distinción entre ambos complementos como inexistente desde un punto de vista estrictamente comunicativo ${ }^{3}$.

Tesnière (1959) fue el primero en establecer una línea divisoria categórica entre ambos tipos de complementos verbales, basándose en criterios fundamentalmente formales y semánticos que se concretan de la siguiente manera:

- Punto de vista de la forma: los actantes vienen representados por sustantivos, frente a los circunstantes que se manifiestan a través de adverbios o equivalentes (preposición + sustantivo).

3 En conexión con este último aspecto, resultan de interés las reflexiones de Vater (1978) y de Adamzik (1992), para quienes la diferenciación entre actantes y circunstantes tampoco resulta significativa si el fin perseguido es dotar a los hablantes de instrumentos eficaces para la producción de textos. "[...] ist es für den Sprecher/Schreiber, der Valenzlexika als Hilfsmittel bei der Textproduktion benutzt, irrelevant, ob bestimmte Konstituenten als Ergänzung oder als Angaben aufzufassen sind. Wichtig ist für ihn, welche Kontextpartner (im Sinne semantische Rollen) typischerweise vorkommen bzw. vorkommen können und wie sie morphologischsyntasktisch realisiert werden". (Adamzik 1992: 310) 
- Punto de vista del sentido: los actantes resultan indispensables para completar el sentido de la predicación, mientras que los circunstantes resultan esencialmente facultativos ${ }^{4}$.

Así, por un lado, Tesniére (1959) sostiene la obligatoriedad de los complementos valenciales o actanciales, como participantes directos del proceso, en contra de la naturaleza facultativa y marginal de los circunstantes, que establecen el marco y las circunstancias en que se desenvuelve la acción $y$, por otro, afirma que esas diferencias de contenido que existen entre uno y otro tipo de complemento se evidencian también a través de determinadas marcas formales de orden morfosintáctico.

De este modo, para el lingüista francés solo Sujeto, CD y CI representarían a elementos configuradores de la valencia verbal, mientras que en el grupo de los circunstantes quedarían englobados, por el contrario, el C. Suplemento o Régimen y todos los complementos circunstanciales. Esta separación tajante entre actancia y circunstancia realizada por Tesnière resulta, a priori, nítida y operativa. Sin embargo, en la práctica dejaba entrever importantes deficiencias que hacían que se mostrara ineficaz y confusa. Incluso el propio lingüista francés reconoció la dificultad para establecer criterios que diferenciaran sin ambigüedad los elementos argumentales de los marginales en la combinatoria verbal. Los desarrollos posteriores que adquirió la TV en los países europeos -sobre todo, en Alemania- heredaron la problemática en torno a la delimitación actancia-circunstancia no resuelta con total solvencia por Tesnière.

Uno de los principales escollos que ha impedido a los investigadores instaurar una frontera perfectamente delimitada entre los componentes actanciales y los circunstanciales tiene que ver con la utilización prioritaria, en la determinación de la actancia, del criterio sintáctico-semántico de obligatoriedad. El empleo de este principio delimitador implica que han de considerarse actanciales complementos habituales que presentan los verbos en determinados contextos, aunque estos complementos se correspondan con lo que, tradicionalmente, denominamos circunstantes y no se manifiesten bajo la estructura sintagmática típica de los elementos argumentales (SN):

La semana que viene vamos a la playa Me arrepenti de todo

\footnotetext{
$4 \quad$ A estos criterios puede añadirse también el denominado nocional que se deduce de las definiciones iniciales y que distingue las entidades que participan en el proceso frente a las circunstancias en que este se desarrolla.
} 
Ambos elementos oracionales constituyen complementos obligatorios del verbo (imposibilidad de supresión), si bien formalmente no se ajustan a las características exigidas por este tipo de componentes, pues constituyen sintagmas preposicionales.

Otro de los problemas que ha surgido en la separación de ambos tipos de constituyentes viene motivado por el hecho de que elementos formalmente idénticos pueden corresponderse unas veces con actantes y otras con circunstantes, incluso con un mismo verbo como núcleo predicativo:

\section{Reside en Murcia (actante)}

Hicimos el examen de conducir ayer en Murcia (circunstante)

Estos fenómenos provocaron la reconsideración de las posturas tesnerianas en los ulteriores desarrollos de la TV. Fue dentro de la lingüística teutona, sin embargo, donde las propuestas valenciales del lingüista francés lograron mayor arraigo, difusión y relevancia: los investigadores alemanes marcaron la hoja de ruta en el devenir de los estudios valenciales que acogieron el resto de países -fundamentalmente Francia y España ${ }^{5}$.

Las interrogantes lanzadas por Tesnière a la palestra de la discusión lingüística trataron de ser respondidas por investigadores procedentes de las dos Alemanias a través de una profusa y fecunda bibliografía que arrancó a finales de los años 60 y principios de los 70 y aún hoy continúa en plena vigencia. Los trabajos de Helbig y Schenckel (1969), Sommerfeldt y Schreiber (1974, 1977), Heringer (1996), Heger (1974), Bondzio (1976, 1977, 1978), Engel y Schumacher (1978), Bossong (1982, 1998), Schumacher et al. (2004), Welke (1988), Wotjak $(1975,1979,1984,1996,2006)$ y Ágel (2000) constituyen solo una pequeña, pero significativa, muestra del enorme interés y desarrollo adquirido por la TV en la literatura germana.

Pese a que no se ha llegado a un consenso unánime en torno al concepto de valencia verbal, la mayor parte de los trabajos surgidos en el ámbito de la TV en el ámbito alemán coinciden en definirla como la capacidad de complementación o combinatoriedad condicionada fundamentalmente desde la semántica, aunque su expresión se realiza desde la sintaxis; es decir,

\footnotetext{
5 En Francia, podemos destacar los trabajos de Lazard (1994, 1998) y Gross $(1975,1981$, 1986). Por su parte, en España resultan relevantes en el ámbito de la TV las investigaciones de Trives (1979), Báez (1988, 2002), Rojo (1990) García-Miguel, 1995a, 1995b). Estos investigadores trataron igualmente de subsanar las deficiencias del planteamiento inicial tesneriano, si bien, para ello, siguieron, esencialmente, la estela de los estudios valenciales de la lingüística teutona.
} 
que la valencia aparece configurada, preferentemente, como una propiedad sintáctico-semántica ${ }^{6}$.

La constatación de la existencia de esa motivación semántica de la valencia llevó pronto a los investigadores a la necesidad de estirar los delgados límites valenciales, así como a caracterizarla como fenómeno no solo sintáctico, sino también lógico-semántico y pragmático: "Tatsächlich gibt es eine prinzipielle Beziehung zwischen der Bedeutung eines Verbs und seinen Mitspielern" (Welke 1988: 99).

Las carencias de algunas de las reflexiones de Tesnière en relación con la delimitación fijada entre los distintos integrantes del predicado verbal hicieron que sus herederos en la lingüística alemana superaran enseguida los criterios formales y semánticos postulados por el lingüista francés para la diferenciación de actantes y circunstantes y se orientaran, a su vez, hacia un concepto ampliado de valencia verbal, basado fundamentalmente, en el principio de indispensabilidad u obligatoriedad sintáctica (Helbig y Schenkel 1969; Helbig 1992) y/o semántica (Bondzio 1976, 1977, 1978; Wotjak 1984): "Nicht nur Subjekte, Objekte und Prädikativa, sondern auch bestimmte Typen von Adverbialbestimmungen bei einigen Verben syntaktisch notwendig sind" (Helbig y Schenkel 1969: 41).

Dada la enorme complejidad en la diferenciación y en aras del establecimiento de unos límites claros se idearon, además, sin éxito, numerosos tests (de índole preponderantemente sintáctica) para el reconocimiento de los argumentos ${ }^{7}$, aunque ninguno de ellos se reveló plenamente eficaz o infalible en la delimitación ${ }^{8}$.

Con objeto de solventar las serias dificultades que aparecían en la delimitación actancia-circunstancia, diversas fueron las soluciones adoptadas por los investigadores alemanes a la hora de atajar el problema. Así, hubo quienes abogaron por efectuar mayores especificaciones dentro de las categorías de argumento y adjunto, aludiendo a la necesidad de diferenciar entre actantes obligatorios, actantes facultativos, adjuntos obligatorios y

\footnotetext{
6 Así viene definida la valencia en el VALBU (2004: 25): "Der Valenzbegriff, der hier zugrunde Wort, hat eine semantische und eine syntaktische Dimension".

7 Los más importantes (cf. Helbig 1992: 78-87), utilizados tanto por Helbig y Schenkel (1969) como por Engel y Schumacher (1978) y puestos en cuestión por Welke (1988), son el de eliminación o test de reducción (Si se elimina el elemento y la oración es agramatical $=$ argumento), el test de implicación semántica del verbo (comer implica algo-alimento-) y el test de conexión y (añadiendo y eso lo hizo).

8 Estas pruebas se mostraban claramente subjetivas, ofrecían resultados contradictorios (por la existencia de contraejemplos) y precisaban del auxilio de criterios semánticos que fijaran la distinción con más precisión.
} 
adjuntos libres (Helbig y Schenckel 1969: 33; Welke 1988: 46). Otros, por el contrario, negaron la existencia de la diferenciación actante-circunstante en virtud de criterios didácticos y comunicativos (Engel y Schumacher 1978: 29; Vater 1978; Adamzik 1992: 310). Finalmente, algunos estudiosos dentro de la lingüística alemana optaron por definir la valencia en términos graduales, rompiendo con la concepción dicotómica tesneriana inicial (Schumacher et al. 2004: 26). De este modo, se entendía que los componentes oracionales podían manifestar diversos grados de vínculo con respecto al núcleo verbal en un abanico que iba desde lo más nuclear a lo más periférico, sin que fuera posible establecer, de acuerdo con estos planteamientos, criterios claros que establecieran cortes netamente definidos en la expansión gradual de la combinatoria verbal.

\section{LA DEFINICIÓN DE VALENCIA EN LA NGLE: LOS COMPLEMENTOS ARGUMENTALES Y LOS ADJUNTOS}

La nueva preceptiva gramatical publicada por la RAE en 2009 constituye la primera gramática del ámbito hispánico que aborda, explícita y profundamente, los planteamientos valenciales y la problemática en torno a la diferenciación entre actantes y circunstantes 9 . Concretamente, la NGLE define el concepto de valencia como "el número de argumentos de un predicado" (RAE 2009: 67). En este sentido, podemos apuntar que la noción de valencia/estructura argumental excede en el texto académico los límites del verbo ${ }^{10}$, pues se considera que también otras unidades lingüísticas (sustantivos, adjetivos y preposiciones, fundamentalmente) poseen virtualidad predicativa: "los predicados no son solo verbales. También los sustantivos, los adjetivos y las preposiciones pueden tener argumentos, es decir, constituyentes exigidos por su significado léxico" (RAE 2009: 64-65).

\footnotetext{
9 Con todo, la impronta del lingüista francés empezó a hacerse visible en las gramáticas del español antes de la aparición de la NGLE, sobre todo en los tratados gramaticales de publicación más reciente. Se observa, no obstante, que las referencias a las ideas tesnerianas se reflejan siempre a través de tímidas incursiones en la noción de valencia que se encuentran íntimamente ligadas a los conceptos de transitividad, complementación y rección (Alcina y Blecua 1975; Alarcos [1994] 2009; Gómez Torrego 2007, Bosque y Demonte 1999, etc.).

10 Tal como apuntara premonitoriamente Tesnière y tal como fuera concebido también, sobre todo, en los desarrollos de la TV en la lingüística alemana.
} 
La presencia de los planteamientos valenciales se hace notar, asimismo, en la preceptiva gramatical académica porque también se asume como válida la clasificación de la tipología de los predicados verbales en función de la valencia -que ya estableciera Tesnière (1994)-. Así, la NGLE clasifica los predicados en función del número de argumentos que se exigen y habla de verbos avalentes, monovalentes, bivalentes y trivalentes (RAE 2009: 67). Por otro lado, aunque, como hemos visto, la cuestión de la delimitación de la frontera entre la actancia y la circunstancia no está enteramente resuelta en el ámbito lingüístico, la NGLE (RAE 2009), siguiendo la tradición valencial, efectúa una división genérica entre dos tipos de complementos, en virtud del vínculo semántico y sintáctico que estos mantienen con respecto al núcleo verbal del que dependen en la cláusula. Así, el tratado académico distingue entre:

- Argumentos o actantes: participantes predicacionales seleccionados semánticamente por el núcleo verbal.

- Adjuntos o circunstantes: participantes predicacionales no exigidos semánticamente por el núcleo verbal.

Tal como podemos observar, ha acabado imponiéndose, en el planteamiento gramatical académico, a la hora de diferenciar los componentes predicacionales, la utilización de un criterio semántico y nocional, lo que supone una amplia superación del criterio formal desarrollado por Tesnière, que fue asumido en líneas generales en la tradición gramatical hispánica. Numerosos pasajes de la NGLE apuntan hacia la motivación semántica de la valencia: los argumentos son complementos necesarios para expresar el significado del verbo ${ }^{11}$, mientras que los adjuntos no vienen exigidos por el verbo y representan especificaciones marginales y de carácter accesorio dentro de la predicación ${ }^{12}$ :

11 En concreto, se habla (RAE 2009: 64) de los argumentos de un predicado verbal como "esqueleto de su significación".

12 El menor grado de interdependencia sintáctica y semántica de los adjuntos con respecto al núcleo verbal del que dependen propicia que, muchas veces -aunque no en todos los casos-, puedan ser suprimidos en la predicación sin que, por ello, se resienta su gramaticalidad. Existen, no obstante, numerosos casos en los que la idiosincrasia de algunos verbos hace que puedan ser eliminados también elementos actanciales en sus predicaciones sin que los predicados dejen de cumplir con las exigencias gramaticales del español: Dibujé vs. Dibujé una casa. 
"Los predicados son categorías que designan estados, acciones, propiedades o procesos en los que intervienen uno o varios PARTICIPANTES. [...] Los participantes seleccionados o elegidos por cada predicado en función de su significación se denominan ARGUMENTOS" [...] Además de estos complementos, se reconocen en la tradición gramatical otros que aportan informaciones no exigidas por ellos. Esos otros complementos se llaman ADJUNTOS, término que equivale a "modificador no seleccionado". (RAE 2009: 66)

Más adelante (RAE 2009: 2908), la preceptiva gramatical vuelve a insistir en la consideración de que la semántica determina la tipología de los complementos que se sitúan en la órbita predicacional:

Se suelen denominar adjuntos los modificadores no seleccionados o no exigidos - casi siempre optativos- que inciden sobre las categorías léxicas y los grupos sintácticos que estas forman. El término adjuntos se usa por oposición al de argumentos, es decir, a los segmentos sintácticos seleccionados o requeridos por un predicado en función de su propia significación.

Sin embargo, pese a que la idea de que los argumentos están sujetos a limitaciones de naturaleza semántica -y ese es uno de los rasgos esenciales que los separan de los adjuntos- planea a lo largo de todo el texto académico $^{13}$, hemos de precisar que la motivación semántica no es privativa de los complementos actanciales. De acuerdo con la NGLE, los adjuntos también sufren restricciones semánticas, esto es, los circunstantes no puedan combinarse con todo núcleo predicativo, pues "no se añaden libremente a cualquier predicado ${ }^{14}$ '. (RAE 2009: 2912)

13 Se considera, en general, (RAE 2009: 2912) que "la distinción entre argumentos y adjuntos se basa $[\ldots]$ en el hecho de que los primeros son complementos seleccionados léxicamente por un predicado, mientras que los segundos se consideran modificadores optativos que agregan particularidades de individuos o de las situaciones".

14 Ya Trives (1979) advirtió que los adjuntos no gozaban de libre combinatoriedad, sino que poseían restricciones de carácter clasemático-genérico. 


\section{LA DELIMITACIÓN ACTANCIA-CIRCUNSTANCIA EN LA NGLE: FUNCIONES SINTÁCTICAS ARGUMENTALES Y CIRCUNSTANCIALES SEGÚN LA RAE}

Como consecuencia del giro semántico acometido en la obra académica, el concepto de valencia que emana de las reflexiones que aparecen en la NGLE resulta más abierto y amplio que el que se deducía en la tradición lingüística hispánica un tiempo atrás. Así, elementos habitualmente considerados como no argumentales -tales como los locativos, los complementos finales o los de cantidad-pasan a integrarse en la órbita valencial de determinado tipo de predicados $^{15}$ :

La mayor parte de los complementos de lugar son adjuntos (Escribió el libro en la mesa), pero algunos son argumentales (como en Puso el libro en esta mesa), en cuanto que están exigidos por la significación verbal. También la mayor parte de los complementos de finalidad son circunstanciales, pero unos pocos se consideran argumentales, ya que están exigidos por el significado del predicado al que modifican, como en Bastaba una leve música para dormirlo. (RAE 2009: 66)

En este sentido, la línea que traza la NGLE para separar argumentos y adjuntos no resulta fija y rígida, sino que se muestra más bien elástica y flexible. La anexión a uno u otro subtipo de complemento predicacional se justifica siempre, en consonancia con los presupuestos anteriormente establecidos, por razones de naturaleza semántica, esto es, en virtud de las exigencias o restricciones impuestas por la significación del núcleo predicativo del que dependen estos elementos. A continuación, revisaremos cuáles son los elementos que integran cada categoría de acuerdo con la postura que sostiene la Academia cuando, a propósito de determinados tipos de unidades sintácticas, menciona su adscripción, bien al subconjunto de los argumentos, o bien al subconjunto de los adjuntos.

Siguiendo la nomenclatura tradicional, según la RAE (2009) los argumentos o actantes se corresponderían con las funciones de Sujeto, CD, Suplemento (C. Régimen), determinados CI y ciertos CC. Los circunstantes

15 Las razones que impulsan este aperturismo son de índole semántica. Ello hace posible que elementos formalmente iguales unas veces se correspondan con argumentos y otras con adjuntos. 
o adjuntos, por su parte, se vincularían con las siguientes funciones: algunos $\mathrm{CC}$, ciertos CI, determinados predicativos y el C. Agente ${ }^{16}$.

\begin{tabular}{|l|l|l|l|}
\hline \multicolumn{2}{|l|}{ Funciones argumentales } & \multicolumn{2}{|l|}{ Funciones no argumentales (adjuntos) } \\
\hline Siempre actantes & $\begin{array}{l}\text { No siempre } \\
\text { actantes }\end{array}$ & $\begin{array}{l}\text { Siempre } \\
\text { circunstantes }\end{array}$ & $\begin{array}{l}\text { No siempre } \\
\text { circunstantes }\end{array}$ \\
\hline $\begin{array}{l}\text { Sujeto } \\
\text { CD } \\
\text { Suplemento (C. } \\
\text { Régimen) }\end{array}$ & $\begin{array}{l}\text { CI } \\
\text { CC Agente }\end{array}$ & $\begin{array}{l}\text { C C , C I , } \\
\text { Predicativos }\end{array}$ \\
\hline
\end{tabular}

Tabla 1. Funciones argumentales y no argumentales según la NGLE (terminología tradicional)

De la información extraída del texto académico puede desprenderse quizá mayor "estabilidad" dentro de las funciones argumentales, estabilidad que contrasta con la alternancia y la versatilidad de los adjuntos, ya que solo una función parece identificarse inequívocamente con esta categoría: la de C. Agente ${ }^{17}$.

Aparecen, como vemos, ciertos desajustes en torno a la pertenencia a uno u otro subgrupo, ya que no se da una correspondencia 1:1 entre argumento y función o entre adjunto y función ${ }^{18}$. Así, parece no haber dudas a la hora de

16 El caso de la función Atributo es peculiar, pues se considera que en las cláusulas con verbos copulativos es la expresión predicativa que ejerce de atributo la que selecciona los argumentos en la oración y no el verbo (por la carga léxica reducida que comporta). (RAE 2009: 2836). En relación con la función Atributo, es preciso aclarar, además, que la RAE (2009: 2774) engloba bajo esta denominación distintos tipos de funciones: predicados de oraciones copulativas, predicados de oraciones semicopulativas, complementos predicativos, predicado de una cláusula absoluta, predicado de una exclamativa bimembre, predicado de otras construcciones no verbales, si bien utiliza la distinción tradicional atributo-predicativo.

17 La RAE incluye los complementos agentes dentro de la categoría de los adjuntos (2009: 2938). En cualquier caso, el texto académico subraya la singularidad de este tipo de complementos, debido a la situación paradójica que les afecta: se presentan sintácticamente como adjuntos, pero semánticamente se corresponden con argumentos del núcleo verbal del que dependen: "El segmento subrayado en Los reos fueron condenados por el juez puede omitirse y supera además las pruebas que identifican complementos circunstanciales [...] Sin dejar de ser adjunto, introduce cierta información que corresponde a un argumento del verbo condenar, concretamente el que designa la persona que lleva a cabo esa acción.

18 La catalogación que efectúa la RAE a propósito de las unidades sintácticas que se integran en cada una de las dos macroclases resulta, a priori, confusa, pues podemos observar cómo algunos elementos oracionales se encuentran ubicados en ambas categorías. Esto sucede 
calificar como argumentos las funciones de Sujeto, $\mathrm{CD}^{19} \mathrm{o}$ los complementos de régimen preposicional ${ }^{20}$. Sí se plantean, por el contrario, ciertas reservas a la hora de otorgar el rango de actante al resto de funciones sintácticas tradicionales.

En la intersección entre argumentos y adjuntos la NGLE sitúa algunos complementos que podemos considerar como problemáticos, dada la dificultad para adscribirlos de forma categórica a una de las dos macroclases de componentes predicacionales. La nueva gramática, asumiendo el desafío de la tradición investigadora en materia valencial, señala que estos elementos dudosos ${ }^{21}$ se corresponden, esencialmente, con los complementos indirectos, los locativos y los predicativos. Tal como había sido comprobado en numerosos trabajos previos -vinculados o no con el modelo valencial-, ninguna de estas categorías funcionales encaja a la perfección en la diferenciación entre argumentos y adjuntos, pues, en virtud de razones de naturaleza eminentemente semántica, unas veces se vinculan con la categoría de los actantes, mientras que otras quedan caracterizados como circunstantes $^{22}$.

porque, tal como hemos explicado anteriormente, la inclusión en el subconjunto de actantes o circunstantes no responde a las características del complemento o la función en sí misma, sino que se vincula específicamente con los rasgos semánticos del verbo del que depende el componente oracional en cuestión.

19 En la NGLE (RAE 2009: 2591), queda establecido que "los complementos directos son elementos argumentales".

20 La argumentalidad de los complementos de régimen preposicional tampoco es puesta en entredicho (RAE 2009: 2715): "Los complementos de régimen preposicional se corresponden, por su parte, con los grupos preposicionales argumentales que están pedidos o seleccionados semánticamente por diversos verbos, sustantivos y adjetivos". Sin embargo, se indica en el texto académico que no siempre resultan obligatorios (Id. 2717) y se efectúan, asimismo, ciertas precisiones en torno a la espinosa diferenciación de este tipo de complementos en relación con otras funciones sintácticas próximas (complemento directo, sujeto, complemento indirecto y, particularmente, el complemento circunstancial y los locativos), advirtiendo de que se trata de una cuestión aún no resuelta en la investigación lingüística actual (Id. 2721 y ss). Estos complementos han sido a veces relacionados en la tradición gramatical hispánica con los $\mathrm{CD}$, debido a la proximidad semántica que manifiestan en algunos ejemplos y a la naturaleza argumental o actancial de ambos. Las semejanzas entre CD y Régimen llevaron incluso hasta el extremo de considerarlos como invariantes de una misma variante funcional (Demonte 1989: 95; Cano Aguilar 1981: 361). Con todo, las diferencias formales existentes entre uno y otro tipo de complemento -Suplemento: S. Preposicional, conmutación por pronombres tónicos; $\mathrm{CD}$ : SN y conmutación por pronombres átonos-y sobre todo la posibilidad de coaparición de Suplementos y $\mathrm{CD}$ en la misma oración han hecho que se les atribuyan estatutos funcionales distintos en el marco oracional.

${ }_{21}$ Se cuestiona (RAE 2009: 2865), además, el estatus de la función de atributo.

22 En este sentido, se alude siempre a las propiedades semánticas idiosincrásicas que presenta cada verbo. 
Así, en relación con el CI, la RAE apunta: "hay complementos indirectos seleccionados, argumentales o actanciales y complementos indirectos no seleccionados, no argumentales o no actanciales, cuyo papel semántico no es central en la predicación" (2009: 2656). Además, se especifica también en el texto académico (Id. 2683-2695) con qué clase de participantes se identifican los CI argumentales y con qué tipo de verbos aparecen combinados ${ }^{23}$ :

\begin{tabular}{|c|c|l|}
\hline \multirow{2}{*}{ CI Argumentales } & Participantes semánticos & \multicolumn{1}{c|}{ Tipos de verbos } \\
\cline { 2 - 3 } & $\begin{array}{l}\text { Destinatarios, experimen- } \\
\text { tadores, complementos de } \\
\text { término, origen y ubicación }\end{array}$ & $\begin{array}{l}\text { Transferencia, comunica- } \\
\text { ción, demanda, intercambio, } \\
\text { asignación, mostración, psi- } \\
\text { cológicos, aproximación, } \\
\text { igualdad, adición, sujeción, } \\
\text { advenimiento, separación, } \\
\text { etc. }\end{array}$ \\
\hline
\end{tabular}

Tabla 2. CI argumentales: participantes semánticos y tipos de verbos

Algo similar sucede en relación con los predicativos (Id. 2865 y 2872-2873). Constituyen complementos limítrofes, pues se identifican unas veces con componentes argumentales y otras con componentes no argumentales de la cláusula ${ }^{24}$.

Según la RAE (2009: 2836 y 2873), los predicativos que aparecen combinados con verbos plenos parecen adscribirse a la categoría de los adjuntos, pues las expresiones predicativas pueden ser omitidas sin que se resienta el significado del verbo: "Compró ilusionado las entradas" vs. "Compró las entradas". En estas dos oraciones el verbo comprar significa exactamente lo mismo: "el verbo no ve alterada su significación ni pierde la capacidad de seleccionar sus argumentos" (Id. 2836). Sin embargo, los predicativos que aparecen en las cláusulas de verbos semicopulativos son "imprescindibles" para expresar el significado del verbo y, por ello, en caso

23 Se señala, asimismo, que los complementos indirectos se corresponden con los dativos de interés, los dativos simpatéticos o posesivos, los dativos éticos y los dativos aspectuales o enfáticos (RAE 2009: 2695 y ss.).

24 En relación con la función "Atributo", es preciso aclarar que la RAE (2009: 2774) engloba, bajo esta denominación, distintos tipos de funciones: predicados de oraciones copulativas, predicados de oraciones semicopulativas, complementos predicativos, predicado de una cláusula absoluta, predicado de una exclamativa bimembre, predicado de otras construcciones no verbales. 
de que no figuren se modifican las propiedades sémicas del verbo: Encuentro raro a Luis vs. Encuentro a Luis ${ }^{25}$.

En este sentido, el texto académico subraya, además, la especificidad semántica de los predicativos en relación con determinados verbos, al percatarse de la existencia de una marcada tendencia a construir con esta clase de complementos las cláusulas de los verbos de juicio y percepción, tales como ver, encontrar, considerar, estimar, creer, juzgar, etc. (RAE 2009: 20875-2876) ${ }^{26}$.

Finalmente, por lo que se refiere a los complementos circunstanciales, la NGLE, siguiendo los parámetros teóricos de la TV, advierte que no todos los elementos oracionales de carácter temporal, local, modal, etc. muestran idénticas características ni ofrecen el mismo comportamiento sintácticosemántico en la cláusula. Por ello, incorpora algunas diferenciaciones habitualmente no tenidas en consideración en el ámbito gramatical. El texto académico constata que determinados complementos que solían integrar la categoría de los circunstantes no encajan con la definición tradicional de adjunto, esto es, no todos constituyen "modificadores optativos" de la cláusula que especifican "particularidades de los individuos o de las situaciones" (RAE 2009: 2912).

Así, de acuerdo con la RAE, cabe efectuar una distinción entre los complementos circunstanciales propiamente dichos -que deben ser incorporados a la categoría de adjuntos ${ }^{27}$ - y otros elementos oracionales seleccionados léxicamente que aportan especificaciones temporales, modales, locales, etc. a determinado tipo de verbos - que se corresponderían con complementos plenamente argumentales-. En efecto, no todos los complementos circunstanciales proporcionan en la cláusula información

25 La RAE (2009: 2873) puntualiza, además, que la distinción seleccionado-no seleccionado no suele aplicarse a los predicativos del sujeto, ya que estos se muestran siempre potestativos y opcionales.

26 El texto académico (RAE 2009: 2877) también apunta a la existencia de predicativos seleccionados en verbos que denotan elección (elegir, proclamar), reconocimiento (confesar, declarar) y denominación (denominar, llamar, nombrar), si bien estos últimos se construyen con predicativos nominales.

27 Algunos de los complementos tradicionalmente considerados circunstanciales pueden ser tipificados efectivamente como componentes marginales no específicamente seleccionados por los núcleos verbales de los que dependen. En esos casos, estos componentes aportan información compatible con el predicado verbal, pero no esencial para el significado de la oración y, por ello, son en muchos casos "optativos".

Dibujé un balón (en la pizarra).

Preparo el cocido (rápidamente).

(Anoche) trabajé en el proyecto. 
fundamentalmente "accesoria" o "residual". Algunos de los elementos oracionales tradicionalmente considerados como circunstanciales vienen exigidos por el verbo en la predicación, por lo que no deberían ser adscritos a la categoría de los adjuntos en sentido estricto ${ }^{28}$. Concretamente, la NGLE reconoce el estatuto diferencial de los argumentos locativos con verbos de movimiento y situativos (ir, residir), los argumentos de manera con verbos de estilo (portarse, actuar) y los argumentos cuantitativos de los verbos de peso, medida duración y precio (costar, medir, durar) (RAE 2009: 2913).

Se trata, en su mayoría, de especificaciones temporales, locales y seleccionadas semánticamente por el verbo que funciona como núcleo predicacional y, por tanto, ostentan un carácter argumental:

\section{Vamos al cine \\ Me comporté correctamente Resido en Murcia}

En este sentido, hemos de apuntar que la NGLE parece abogar por la introducción de una nueva categoría funcional capaz de dar cuenta de las peculiaridades que presentan específicamente este tipo de componentes oracionales.

Especial hincapié hace el texto académico en los complementos locativos. De este modo, a pesar de que reconoce que resulta dudosa su caracterización como argumentos en determinadas circunstancias, en general, considera conveniente establecer distinciones con respecto al comportamiento que manifiestan los componentes situativos en cláusulas con verbos como caber, pernoctar, residir, sobre la base de ciertos rasgos que permiten diferenciarlos del resto de funciones.

Para justificar la creación de esta nueva categoría funcional en el orden sintáctico, que en el texto académico se denomina complemento argumental de lugar, la NGLE (RAE 2009: 2723) utiliza los mismos criterios diferenciadores apuntados por $\operatorname{Rojo}^{29}(1985,1990)$ a la hora de separar los complementos que él denomina adverbiales de los suplementos:

28 Estos complementos se identifican con elementos tradicionalmente considerados como circunstantes que, al estar seleccionados léxicamente, se comportan más propiamente como actantes o argumentos en relación con el núcleo verbal del que dependen.

29 Ante las insuficiencias existentes en la caracterización de los complementos circunstanciales investigadores como Rojo $(1985,1990)$ propusieron la introducción de una nueva categoría funcional en el orden sintáctico: la función de complemento adverbial. Bajo este marbete, Rojo incluyó elementos predicacionales de muy diversa naturaleza: a) C. Adv. 1: complementos locativos que acompañan a verbos de movimiento o de situación; b) C. Adv. 
1) conmutación por adverbios y 2) no rección preposicional (posibilidad de alternar distintas preposiciones) $)^{30}$.

Así, conforme a lo que hemos establecido y de acuerdo con las nuevas etiquetas terminológicas que aparecen en el texto académico, la NGLE establece la siguiente distribución entre funciones argumentales y circunstanciales.

\begin{tabular}{|c|c|}
\hline $\begin{array}{c}\text { Funciones argumentales } \\
\text { (actantes) }\end{array}$ & $\begin{array}{c}\text { Funciones no argumentales } \\
\text { (adjuntos o circunstantes) }\end{array}$ \\
\hline $\begin{array}{l}\text { Sujeto } \\
\text { CD } \\
\text { Suplemento (C. Régimen) } \\
\text { Complementos argumentales de lugar } \\
\text { (verbos de movimiento y situativos) } \\
\text { Argumentos cuantitativos (verbos de } \\
\text { peso, medida, duración y precio) } \\
\text { Argumentos de manera (verbos de estilo) } \\
\text { Algunos CI (distintas clases semánticas } \\
\text { de verbos) } \\
\text { Algunos Predicativos (verbos semicopu- } \\
\text { lativos y verbos de juicio y percepción) }\end{array}$ & $\begin{array}{l}\text { C. Agente } \\
\text { CC } \\
\text { Algunos CI } \\
\text { Algunos Predicativos }\end{array}$ \\
\hline
\end{tabular}

Tabla 3. Funciones argumentales y no argumentales según la NGLE (nueva terminología)

2. complementos cuantitativos de verbos de peso, medida, duración y precio; c) C. Adv. 3. complementos modales de verbos de estilo (actuar, portarse).

Aunque los planteamientos de Rojo fueron criticados fundamentalmente por la heterogeneidad de elementos que configuraban la nueva categoría funcional (Pérez 1989: 477-478), este investigador justificó la creación de una nueva etiqueta sintáctica $(1985,1990)$, a través de los siguientes argumentos: 1) Se trata de complementos nucleares; 2) admiten la conmutación por adverbios; 3) constituyen complementos cuyo significado muestra congruencia con el contenido léxico del verbo que los exige.

30 La consideración de los complementos situativos como componentes argumentales en virtud de las propiedades sémicas específicas de determinados verbos (verbos estativos y de movimiento) procede de la tradición valencial (Helbig) y del enfoque funcionalista (Dik 1981). En el ámbito hispánico, destacan, en este sentido, además de las aportaciones de Rojo (1990), los planteamientos de Gómez Torrego (2007) que habla de argumentos adverbiales, y de Trives (1979: 199-210; 1982: 147-148 y 173-179) y Cifuentes (1988-1989: 199). Estos dos últimos investigadores ofrecen la distinción entre verbos locales y los verbos localizables: "Llamaremos, pues, verbos locales o locativos a aquellos cuyo semismo exige específicamente -en un determinado esquema un complemento local, que será considerado actante. [...] Verbos localizables serán aquellos que tan solo suponen de forma genérica o clasemática un comportamiento local, siendo considerado en este caso como circunstante". (Cifuentes 1988-1989: 150) 


\section{1. MeCANismos DE IDENTIFICACión DE LA ACTANCIA}

Tal y como hemos venido señalando, son muchas las páginas de la NGLE en las que se alude a la dificultad de delimitar actancia y circunstancia.

la oposición entre argumentos y adjuntos [...] se vuelve escurridiza cuando se aplica a ciertos casos conflictivos [...] los criterios delimitativos que suelen aplicarse a esos contextos no proporcionan en todas las construcciones resultados enteramente satisfactorios. (RAE 2009: 2912)

son poco nítidos los límites entre los complementos de régimen y los circunstanciales en bastantes ocasiones. (RAE 2009: 2723)

Aunque muchos gramáticos piensan en la actualidad que la estructura gramatical de las oraciones con complementos locativos argumentales difiere de la que ofrecen los verbos que aceptan complementos circunstanciales de lugar, la distinción se hace escurridiza en ciertos casos. (RAE 2009: 2724)

Ante la problemática adscripción que manifiestan algunas clases de unidades sintácticas bien a la categoría de adjuntos o bien a la de los argumentos, la NGLE (RAE 2009: 2908) proporciona algunas claves con las que se pretende ayudar a identificar con más claridad el vínculo que los une con el núcleo predicativo del que dependen. En este sentido, con objeto de establecer unos límites claros entre actancia y circunstancia, la nueva gramática remite a la utilización de pruebas o tests que ayudan a caracterizar sintácticamente los complementos adjuntos ${ }^{31}$. En concreto, el texto académico menciona la prueba de eliminación, que se basa en la constatación de que la gramaticalidad de la secuencia no suele resentirse ante la ausencia de los modificadores, pero sí ante la omisión de los actantes: "Uno de los rasgos prototípicos de los adjuntos es que su omisión no suele alterar la gramaticalidad de la oración". (RAE 2009: 2914).

Sin embargo, al igual que hicieron con anterioridad los investigadores de la TV, la Academia precisa que el test de eliminación no es una condición necesaria, pues no todos los argumentos resultan obligatorios ${ }^{32}$. Algunos

31 La NGLE (RAE 2009 2914) señala que ante las dificultades que plantea el criterio de la selección léxica, las pruebas sintácticas parecen constituir los métodos más apropiados "en la tarea de distinguir los argumentos de los adjuntos".

32 Se habla concretamente de argumentos implícitos (RAE 2009: 68). En cualquier caso, también se menciona la imposibilidad de supresión de los adjuntos si algún elemento oracional 
de ellos, aunque con mayores restricciones, pueden ser omitidos sin que la cláusula pase a ser agramatical (RAE 2009: 68, 2914).

Tampoco otros sistemas o métodos de reconocimiento, mencionados en la propuesta académica (RAE 2009: 2915-2918) siguiendo en cierta medida la tradición valencial, tales como las interrogativas indirectas truncadas, las perifrasis condicionales o copulativas enfáticas condicionales o la sustitución por la proforma hacerlo, se revelan plenamente eficaces o infalibles en la fijación de la delimitación argumento-adjunto, debido, fundamentalmente, a restricciones léxicas idiosincrásicas (Id. 2918).

Las pruebas sintácticas basadas en la supresión de complementos dependen de un factor inicial de naturaleza estrictamente léxica, lo que limita en alguna medida su eficacia como recursos probatorios o discriminadores en los contextos mencionados.

La ineficacia de los procedimientos de reconocimiento de actantes obliga a la RAE a recurrir a la concepción de la actancialidad como continuum (RAE 2009: 2918). La consideración de la actantancialidad como fenómeno gradual parece aproximar la propuesta de la RAE a la que Hopper y Thompson (1980) realizan con respecto a la transitividad ${ }^{33}$ y también vincula su postura con otros planteamientos efectuados más específicamente en el ámbito de la TV.

La ruptura con la concepción dicotómica tesneriana inicial entre complementos y adjuntos y la evolución de la TV hacia la no consideración del binomio actante-circunstante como oposición binaria se evidencia, por ejemplo, en el marco de la lingüística alemana, en el principio teóricometodológico básico que encontramos en la introducción al último diccionario valencial del alemán, el VALBU:

Die Probleme ergeben sich daraus, dass die Bindungsfestigkeit zwischen Verb und Umgebung nicht nur zwei Stufen aufweist, die eine eindeutige Differenzierung von zwei Klassen von Satzgliedern ermöglichen würde, sondern ein Kontinuum, das verschiedene Grenzziehungen ermöglicht. (Schumacher et al. 2004: 26)

remite específicamente a ellos en la secuencia (RAE 2009: 2914). Con todo, se señala que las condiciones de omisión de los argumentos son mucho más restrictivas que las de los adjuntos.

33 Hopper y Thompson señalan específicamente (1980: 299): "Transitivity is a global property of clauses, that it is a continuum along which various cluster and tend strongly to cooccur, and that the foci of high Transitivity and low Transitivity correlate with the independent discourse notions of foregrounding and backgrounding respectively". Esta postura también es asumida en gran medida por Cano Aguilar (1981) Hernanz y Brucart (1987). 
No obstante, es preciso advertir que, según la NGLE, la naturaleza gradual constituye un rasgo atribuible, en principio, solo a la posibilidad de separabilidad u omisión de los adjuntos y no a la propia oposición argumento-adjunto ${ }^{34}$. Esta consideración contraviene, en cierta medida, lo sostenido desde la mayor parte de las propuestas de los enfoques valenciales que apostaban, ante la imposibilidad de delimitar con exactitud los límites valenciales, por la existencia de una oposición gradual y no binaria entre los dos tipos de complementos predicacionales.

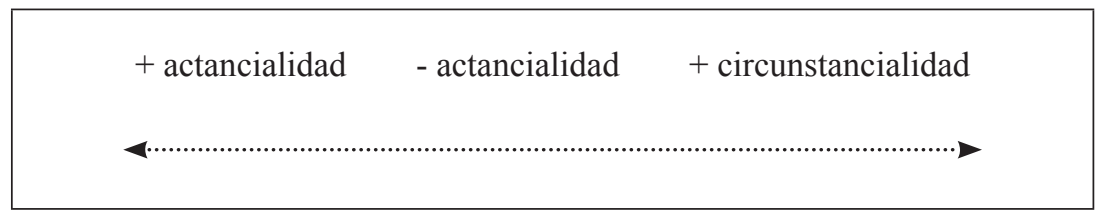

Tabla 4. Actancialidad como continuum.

\section{RECAPITULACIÓN Y CONCLUSIONES}

La publicación de la NGLE supone, tal como hemos visto, la incorporación/ asunción en la reflexión lingüística hispánica de buena parte de los presupuestos valenciales iniciados por Tesnière en 1959 y la indagación

34 En general, muchos autores han optado con mayor o menor explicitud por esta diferenciación gradual en relación con los componentes que configuran un predicado verbal (Ágel 2000; Schumacher et al. 2004; García-Miguel 1995a y 1995b; Trives 1979, etc.) y solo Cano Aguilar (1999) aplica esta gradualidad al nivel de exigencia de actualización discursiva o formalización. En este sentido, también es interesante la aportación de Lazard (1998). De acuerdo con este lingüista, la actancia representa un continuum en el que es preciso establecer una gradación entre actantes centrales, actantes periféricos, adjuntos y circunstantes: "On est conduit à distinguer des actants "centraux" et des actants "périphériques", c'est à dire plus distants du verbe. Les circonstants, qui n’ont pas de lien spécifique avec celui-ci, sont les termes les plus distants. Les adjets, dont le seul lien grammatical avec le verbe est d'être requis, le sont un peu moins". (Lazard 1998: 18). Tras la observación pormenorizada y el análisis exhaustivo del patrimonio lingüístico europeo, Lazard articula una jerarquía entre los complementos verbales, en virtud de la oposición generada entre exigencia (necesidad de formalización) y rección (necesidad sintáctica). Así, este lingüista habla de actantes exigidos y regidos (actantes centrales), de actantes simplemente regidos, pero no exigidos (actantes periféricos o facultativos), de actantes simplemente exigidos (adjuntos) y de complementos ni exigidos ni regidos (circunstantes). 
en el espinoso asunto de la diferenciación actante-circunstante. Aunque las ideas valenciales planean en buena parte del texto académico, es sobre todo en el capítulo dedicado a los adjuntos y en el dedicado a los complementos de régimen preposicional donde más se incide en la diferenciación actantecircunstante.

La profundización en la delimitación de los límites entre argumentos y adjuntos que pretende suplir las deficiencias que muestra la vieja dicotomía transitivo-intransitivo -incapaz de determinar con exactitud cuál es el comportamiento sintáctico de los verbos-constituye un hecho prácticamente inédito en las preceptivas gramaticales del mundo hispánico. Antes de que la RAE alumbrara la NGLE en 2009, la presencia de la TV era nula o muy vaga dentro de las gramáticas del español, incluso aunque se tratara de textos gramaticales no concebidos en el ámbito académico.

Tal como hemos comprobado, la nueva preceptiva gramatical, siguiendo la estela del enfoque valencial, traza una línea divisoria entre dos tipos de componentes predicacionales: los actantes y los circunstantes. Los actantes $\mathrm{o}$ argumentos se distinguen de los circunstantes o adjuntos, en virtud de un criterio distintivo que se revela fundamental: la selección léxica. Los actantes constituyen complementos seleccionados por el lexema verbal que funciona como núcleo de la cláusula: vienen exigidos por las peculiaridades sémicas del verbo y, por ello, normalmente no pueden ser omitidos sin que se resienta la gramaticalidad de la oración. Los circunstantes, por el contrario, se identifican con elementos no seleccionados por el lexema verbal: son componentes significativamente compatibles con el núcleo predicacional, pero su elisión no impide la conformación de un predicado gramaticalmente aceptable.

La definición de la valencia en términos eminentemente semánticos y la profundización en el establecimiento de la frontera entre actancia y circunstancia lleva a la RAE a trazar nuevos caminos y a abrir espacios funcionales en el orden sintáctico hasta entonces prácticamente desconocidos e inexplorados en la reflexión gramatical de corte académico dentro del ámbito de la lengua española.

En virtud del análisis que hemos efectuado, podemos comprobar que, de acuerdo con los planteamientos más recientes de la TV, se produce en el texto académico una superación amplia y expresa del criterio formal planteado inicialmente por Tesnière que dejaba fuera de la órbita valencial a complementos que no tuvieran la estructura de SN. Este cambio de orientación se traduce en que el texto académico reconoce el estatuto argumental de determinado tipo de elementos oracionales tradicionalmente enmarcados en la categoría de adjuntos. 
La línea que separa la actancia de la circunstancia dentro de las páginas de la NGLE no resulta, sin embargo, estática o rígida. Los elementos oracionales parecen no confinarse a las categorías enteramente cerradas, ya que no existe una correspondencia 1:1 entre categoría y función: determinadas funciones se corresponden unas veces con actantes y otras, con circunstantes. En cualquier caso, el texto académico parece apuntar a una mayor especialización funcional de los argumentos frente a los adjuntos. Solamente una función, de acuerdo con la RAE, se adscribe inequívocamente a la categoría de los circunstantes -el C. Agente- frente a las tres que invariablemente se sitúan en la dimensión argumental: Sujeto, CD y C. Régimen.

El resto de las funciones sintácticas oracionales se revelan más controvertidas y particularmente limítrofes. Se trata, en todo caso, de componentes predicacionales habitualmente situados en la tradición valencial en la intersección argumento-adjunto: los predicativos, los CI y los complementos circunstanciales.

Conforme establece la NGLE, solo en determinadas circunstancias los CI y los predicativos constituyen funciones plenamente argumentales dentro de la cláusula. Para que estas funciones posean un estatuto argumental, han de cumplirse ciertos requisitos -fundamentalmente semánticos- en los verbos que actúan como núcleos oracionales. Así, en el caso de los CI, estos serán argumentales únicamente cuando el lexema verbal del que dependan pertenezca a las clases semánticas de transferencia, comunicación, demanda, intercambio, asignación, mostración, psicológicos, aproximación, igualdad, adición, sujeción, advenimiento, separación, etc. Por lo que respecta a los predicativos, su naturaleza argumental vendrá determinada principalmente por la concurrencia de verbos semicopulativos y de verbos de juicio y percepción.

Los CC constituyen, por el contrario, un capítulo aparte, pues, en este caso, no es que a veces estos complementos se comporten como argumentos, sino que ciertos elementos predicacionales que tradicionalmente han sido adscritos a esta categoría deben ser excluidos de ella porque, en realidad, se corresponden con nuevas etiquetas o categorías funcionales. Concretamente, la RAE habla de tres componentes actanciales habitualmente tipificados como circunstantes: los complementos argumentales de lugar, los argumentos cuantitativos y los argumentos de manera. Este tipo de constituyentes también atienden a restricciones semánticas: solo se combinan con verbos de movimiento y verbos situativos, con verbos de peso, medida, duración y precio y con verbos de estilo, respectivamente.

Con todo, hemos de señalar que la elasticidad y la ausencia de rigidez en la delimitación de los actantes que efectúa la NGLE redunda, en ocasiones, en cierta indefinición teórico-práctica. Aunque, como hemos visto, es habitual 
que la NGLE efectúe precisiones en torno a qué verbos o a qué tipo de verbos admiten como argumentos algunos complementos tradicionalmente considerados como adjuntos, no siempre los datos sobre la combinatoria verbal se especifican con detalle en la obra académica, seguramente por la existencia de restricciones léxicas idiosincrásicas que impiden dilucidar los límites entre una y otra categoría con exactitud. Por otro lado, la información suele aparecer de forma bastante fragmentada y deslavazada a lo largo de las páginas del texto académico y, por ello, a veces resulta difícil recuperarla y asimilarla convenientemente. En este sentido, quizá sería útil condensar la información valencial en un único capítulo e incorporar a la gramática una sección o apartado adicional que emule las tablas de palabras construidas con preposición habituales en las ediciones anteriores de las preceptivas gramaticales académicas ${ }^{35}$.

La problemática en torno a la diferenciación entre actantes y circunstantes no queda, por tanto, solventada en el texto académico en términos absolutos, sino que se muestra como un asunto no plenamente cerrado dentro de la reflexión lingüística actual que queda pendiente de resolución o respuesta en la discusión gramatical ${ }^{36}$.

Las fronteras entre una y otra categoría vacilan y son escurridizas, a pesar de que, como ya ocurriera en planteamientos valenciales previos, se recurra a la definición de la argumentalidad como fenómeno gradual para proporcionar una eventual solución a este opaco punto de la propuesta teórica valencial.

La TV, cuyos presupuestos esenciales acostumbraban a figurar en buena parte de los tratados gramaticales más representativos de la lengua española bajo la cáscara de los conceptos de transitividad, rección y complementación, fue hasta 2009 una de las ausencias teóricas más marcadas de la reflexión lingüística hispánica. Su presencia en la tradición de la lengua española es más bien discreta, incluso tras la publicación y la difusión de la influyente obra del lingüista francés Lucien Tesnière en español.

35 En la primera gramática publicada por la RAE en 1771, comienza la que acabaría por convertirse en una larga tradición, tanto en los tratados gramaticales académicos -con excepción de la muy reciente NGLE- como en algunos no académicos -como en la gramática de Salvá de 1831-: la introducción de una larga lista de palabras que se construyen con preposición. El objetivo declarado que persigue la RAE, al incluir este compendio de palabras ordenado alfabéticamente que también incluye ejemplos ilustrativos, no es otro que el de completar "una parte muy esencial de la sintaxis" y el de ayudar a "salir con facilidad de cualquiera duda que ocurra" (RAE 1771: 251).

36 Se precisa (RAE 2009: 2918) que se trata de una cuestión no resuelta en la investigación lingüística actual. 
La aceptación de los principios teóricos y metodológicos del paradigma valencial en la que pasa por ser la opera magna de la reflexión lingüística de las Academias de la Lengua española, supone, por ello, un gran avance. El reflejo de las teorías valenciales en la NGLE constituye, a nuestro juicio, un buen síntoma de la vigencia, valide ${ }^{37}$ y operatividad de este modelo teórico en la explicación del fenómeno lingüístico, a la vez que representa un justo tributo a sus virtudes y a sus méritos, después de décadas de largo silencio.

Así, pese a que existen ciertas carencias y algunos "puntos débiles" 38 dentro de la NGLE, pues no llega a clarificar con total garantía cuándo un elemento se adscribe a las categorías de adjunto o argumento, obligando a recurrir a la intuición en los casos dudosos, es preciso reconocer la importancia y el valor que tiene el giro semantista llevado a cabo en la investigación lingüística académica y apreciar el hecho de que los estudios en torno a la valencia y a la combinatoria verbal hayan encontrado al fin eco y desarrollo en la tradición gramatical hispánica.

\section{REFERENCIAS BIBLIOGRÁFICAS}

AdAmziK, Kirsten. 1992. Ergänzungen zu Ergänzungen und Angaben. Deutsche Sprache 20: 289-313.

Àgel, Vilmos. 2000. Valenztheorie. Tubinga: Gunter Narr Verlag.

Alarcos Llorach, Emilio. [1994] 2009. Gramática de la lengua española. Madrid: EspasaCalpe.

Alcina, Juan y Blecua, José Manuel. 1975. Gramática española. Barcelona: Ariel.

Bondzio, Wilhelm. 1976/1977/1978. Abriss der semantischen Valenztheorie als Grundlage der Syntax. Zeitschrift für Phonetik, Sprachwissenschaft und Kommunikationsforschung 4/1976, 3/1977 y 1/1978: 354-363, 261-273, 21-33.

Bosque, Ignacio y Demonte, Violeta. 1999. Gramática descriptiva de la Lengua española. Madrid: Espasa-Calpe.

Bossong, Georg. 1982. Actance ergative et transitivité. Le cas du système verbal de l'oubykh. Lingua 56, 3-4: 201-234.

37 Otra prueba fehaciente de la gran actualidad de los planteamientos tesnerianos puede observarse en el hecho de que su obra-olvidada e ignorada durante mucho tiempo en el ámbito hispánico- fue traducida y publicada en la editorial Gredos en el año 1994, a pesar de que la primera edición francesa databa, como ya hemos señalado, de 1959.

38 En general, los planteamientos de la NGLE presentan las mismas deficiencias que se atribuyen a los desarrollos actuales de la TV, sobre todo en lo referente a la indefinición del plano en que debe situarse la valencia o al establecimiento de una frontera clara entre actancia y circunstancia. 
1998. Le marquage différentiel de l'objet dans les langues d'Europe en Feuillet, Jack (ed.), pp. 193-258.

CANo Aguilar, Rafael. 1981. Estructuras sintácticas transitivas del español actual. Madrid: Gredos.

1999. Los complementos de régimen verbal. En Ignacio Bosque y Violeta Demonte (eds.), vol. 2, pp. 1807-1854.

Cifuentes Honrubia, José Luis. 1988-1989. Sobre las construcciones locales en español. Estudios de lingüistica 5: 145-181.

Demonte, Violeta. 1989. Teoría sintáctica: de las estructuras a la rección. Madrid: Síntesis. Diк, Simon. 1981. Gramática funcional. Madrid: Sociedad General Española de Librería.

Engel, Ulrich y Schumacher, Helmut. 1978. Kleines Valenzlexikon deutscher Verben. Tubinga: Gunter Narr.

Feuillet, JACK (ED.). 1998. Actance et valence dans les langues de l'Europe. París: Mouton de Gruyter.

García-Miguel, José María. 1995a. Las relaciones gramaticales entre predicado y participantes. Santiago de Compostela: Universidade, Servicio de Publicacións e Intercambio Científico.

-1995b. Transitividad y complementación preposicional en español. Verba. Santiago de Compostela, Anuario Galego de Filología. Anexo 40. Universidade, Servicio de Publicacións e Intercambio Científico.

Gómez Torrego, Leonardo. [1997] 2007. Gramática didáctica del español. Madrid: SM.

Heger, Klaus. 1974. Teoría semántica. Hacia una semántica moderna II. Madrid: Alcalá.

Helbig, Gerhard. 1992. Probleme der Valenz und Kasustheorie. Tubinga: Niemeyer.

Helbig, Gerhard y Schenkel, Wolfgang. 1969. Wörterbuch zur Valenz und Distribution deutscher Verben. Leipzig: VEB Verlag Encyklopädie.

Heringer, Hans Juergen. 1996. Deutsche Syntax dependentiell. Tubinga: Stauffenburg.

Hernanz, María Luisa y José María Brucart. 1987. La sintaxis. Barcelona: Crítica.

Hopper, Paul J. y Thompson, Sandra A. 1980. Transitivity in Grammar and Discourse. Language, 56, 2: 251-299.

Lazard, Gilbert. 1998. Définition des actants dans les langues européennes. En Feuillet, Jack (ed.). Actance et Valence dans les Langues de l'Europe. Berlín-Nueva York: Mouton de Gruyter.

Mendikoetxea, Amaia. 1999. Construcciones inacusativas y pasivas. En Ignacio Bosque y Violeta Demonte (eds.), vol. 2, pp. 1575-1630.

Pérez, María Rosa. 1989. Consideraciones acerca de los complementos adverbiales. Verba: Anuario galego de filoloxia 16: 469-479.

Perlmutter, David M. 1978. Impersonal passives and the Unaccusative Hypothesis. Berkeley Linguistics Society 4: 157-189.

Ramón Trives, Estanislao. 1979. Aspectos de semántica lingüistico-textual. Madrid: Ediciones Istmo-Alcalá. Murcia: Godoy.

Real Academia Española. 1771. Gramática de la lengua castellana. Madrid: Joaquín Ibarra. 2009. Nueva gramática de la lengua española. Madrid: Espasa Libros.

Rojo, Guillermo. 1985. En torno a los complementos circunstanciales. En Lecciones del I y II curso de Lingüística funcional (1983 y 1984), Oviedo, pp. 181-190.

1990. Sobre los complementos adverbiales. En Homenaje al Prof. Francisco Marsá. Barcelona: Universidad de Barcelona, pp. 153-171. 
SOMmerfeldt, KarL-ERnst y Herbert Schreiber. 1974. Wörterbuch zur Valenz und Distribution deutscher Adjektive. Leipzig: VEB Bibliographisches Institut.

1977. Wörterbuch zur Valenz und Distribution der Substantive. Leipzig: VEB Bibliographisches Institut.

Schumacher, Helmut, Jacqueline Kubczak, Renate Schmidt y Vera de Ruiter. 2004. VALBU Valenzwörterbuch deutscher Verben. Tubinga: Narr Francke Attempto.

SALVÁ, Vicente. [1831] 1988. Gramática de la lengua castellana según ahora se habla. Estudio y edición de Margarita Literas. Madrid: Arco Libros

TesniÈRe, LuCIEN. [1959] 1994. Elementos de sintaxis estructural. Madrid: Gredos.

Trives, Ramón. 1979. Aspectos de semántica lingüistico-textual. Madrid: Istmo. 1982. Estudios sintáctico-semánticos del español. Murcia: Godoy.

VAter, Heinz. 1978. Probleme der Verbvalenz. Leuvense Bijdragen Louvain 67, 3: 267-308.

WelKe, KLAus. 1988. Einführung in die Valenz- und Kasustheorie. Leipzig: Bibliographisches Institut.

WotjaK, Gerd. 1975. Semantische Merkmalanalyse und Valenzmodelle. Vox Romanica 34: $1-26$.

1979. Investigaciones sobre la estructura del significado. Madrid: Gredos.

1984. Zur Aktantifizierung von Argumenten ausgewählter deutscher Verben. Zeitschrift für Germanistik 4, pp. 401-414.

Narr Verlag.

(ED.). 1996. En torno al adverbio español y los circunstantes. Tubinga: Gunter

2006. Las lenguas, ventanas que dan al mundo. Salamanca: Servicio de Publicaciones de la Universidad de Salamanca. 